\title{
Role of central and peripheral serotonin in obesity: what to expect in the near pharmacotherapy future?
}

\section{Opinion}

Obesity currently represents a global epidemic disease, amounting for an estimated $\$ 147$ billion in health care spending annually in USA only, and associated with cardiovascular and cerebrovascular diseases. ${ }^{1}$ Regarding the pathogenesis, it's well known that obesity results from an imbalance between energy intake and expenditure, and a hypothesis that is widely accepted states that increased food intake is partially driven by decreased dopamine-mediated reward and decreased serotonin, (5-hydroxytryptamine [5-HT]), mediated homeostatic feedback in response to food intake. ${ }^{2}$ Central serotonergic system is known to elicit satiety signal as well as to elevate the mood and evidence suggested a positive and bidirectional association between obesity and depression. ${ }^{3}$ Interestingly, not only central serotonin plays a role in obesity pathogenesis, but also peripheral serotonin was suggested to play an important role in obesity, and evidence from animal studies has demonstrated 5-HT ability to regulate glucose and lipid metabolism by accelerating energy consumption in skeletal muscle. Further, gut-derived 5-HT was likely suggested to be an important driver of pathogenesis in human obesity as well as in dysglycemia ${ }^{4,5}$ and several genetic polymorphisms in tryptophan hydroxylase and 5-HT receptors were shown to have strong associations with obesity. Moreover, peripheral 5-HT was shown to suppress the adaptive thermo genesis in brown adipose tissue and the peripheral serotonergic system was marked as a therapeutic target for both obesity and diabetes. ${ }^{6}$ Lorcaserin, 5-HT2 receptor agonist, and its potential under development more specific congeners with lower adverse effects, suppress the appetite and reduce body weight gain and are considered as promising therapeutic tools for obesity ${ }^{7}$ while antagonism of 5-HT2c receptor has been identified as a main cause of second generation antipsychotics induced weight gain and obesity. ${ }^{8}$ On the other hand, obesity increases peripheral serotonin and inhibiting serotonin signaling or its synthesis in adipose tissue may be an effective treatment for obesity and its comorbidities. ${ }^{9}$

\section{Acknowledgements}

None.

\section{Conflict of interest}

Author declares that there is no conflict of interest.

\author{
Volume 8 Issue 6 - 2018 \\ Mina T Kelleni ${ }^{1,2}$ \\ 'Department of Pharmacology, Jouf University, Kingdom of Saudi \\ Arabia \\ ${ }^{2}$ Department of Pharmacology, Minia University, Egypt
}

Correspondence: Mina T Kelleni MD, PhD, Department of Pharmacology, College of Medicine, Jouf University, Sakaka, Kingdom of Saudi Arabia, Tel +966560407874 Email drthabatpharm@yahoo.com

Received: July 29, 2018 | Published: November 09, 2018

\section{References}

1. Gomez G, Stanford FC. US health policy and prescription drug coverage of FDA-approved medications for the treatment of obesity. Int J Obes (Lond). 2018;42(3):495-500.

2. Van Galen KA, Ter Horst KW, Booij J, et al. The role of central dopamine and serotonin in human obesity: lessons learned from molecular neuroimaging studies. Metabolism. 2018;85:325-339.

3. Haleem DJ. Drug Targets for Obesity and Depression: From Serotonin to Leptin. Curr Drug Targets. 2016;17(11):1282-1291.

4. Watanabe H, Nakano T, Saito R, et al. Serotonin Improves High Fat Diet Induced Obesity in Mice. PLoS One. 2016;11(1):e0147143.

5. Young RL, Lumsden AL, Martin AM, et al. Augmented capacity for peripheral serotonin release in human obesity. Int J Obes (Lond). 2018.

6. Oh CM, Park S, Kim H. Serotonin as a New Therapeutic Target for Diabetes Mellitus and Obesity. Diabetes Metab J. 2016;40(2):89-98.

7. García-Cárceles J, Decara JM, Vázquez-Villa H, et al. A Positive Allosteric Modulator of the Serotonin 5-HT2C Receptor for Obesity. J Med Chem. 2017;60(23):9575-9584.

8. Huang XF, Weston-Green K, Yu Y. Decreased 5-HT2cR and GHSR1a interaction in antipsychotic drug-induced obesity. Obes Rev. 2018;19(3):396-405.

9. Crane JD, Palanivel R, Mottillo EP, et al. Inhibiting peripheral serotonin synthesis reduces obesity and metabolic dysfunction by promoting brown adipose tissue thermogenesis. Nat Med. 2015;21(2):166-172. 